H I G H L I G H T S

\section{Close encounters}

HIV infection spreads through the closest of human interactions and now, new research shows that the virus can also take advantage of intimate contacts between cells of the immune system to aid infection of its main target, the $\mathrm{CD}^{+} \mathrm{T}$ cell.

Dendritic cells (DCs) are the principal antigen-presenting cells (APCs) that are involved in the initiation of an immune response. They internalize antigens, which are processed and presented to $\mathrm{T}$ cells together with costimulatory signals that are essential for T-cell activation. But it seems that HIV has taken advantage of this essential immune interaction: DCs can bind and internalize HIV virions through C-type lectin receptors but instead of becoming infected, the DCs assist in the infection of T cells. To find out exactly how this happens, David McDonald and co-workers pulsed myeloid-derived DCs with HIV-1, then observed their interactions with T-cell targets using time-lapse fluorescent imaging.

Before T-cell contact, the virus was distributed evenly on or inside the DC, but within minutes most of it had concentrated in the T-cell-DC contact zone. Internalized HIV was also transported to this site. This redistribution is reminiscent of the formation of structured synapses at the contact region between T cells and APCs. T-cell-DC synapses are a special case, in that they can form in the absence of specific antigen - a process that does not lead to activation but might help T cells to 'sniff out' rare antigen-presenting DCs.

Immunostaining of conjugates between $\mathrm{CD}^{+} \mathrm{T}$ cells and HIV-pulsed DCs showed that CD4 and the adhesion molecule LFA1 (leukocyte function-associated antigen 1) - which are both involved in the antigen-independent synapse - as well as the HIV coreceptors CCR5 (CC-chemokine receptor 5) and CXCR4 (CXC-chemokine receptor 4), were often concentrated at the cell-cell junction. In several of these conjugates, HIV was detected inside the T cell, indicating that infection had occurred across the synapse. This process was even more efficient when the DCs were matured with lipopolysaccharide.

Together these observations support the idea that HIV has subverted the antigen-independent synapse for its own ends, which takes our appreciation of immune exploitation by HIV to a whole new level.

Jennifer Bell, Senior Editor, Nature Reviews Immunology

\section{2) References and links}

ORIGINAL RESEARCH PAPER McDonald, D. et al. Recruitment of HIV and its receptors to dendritic cell-T-cell junctions. Science 2003 May 1 (DOI: 10.1126/science.1084238)

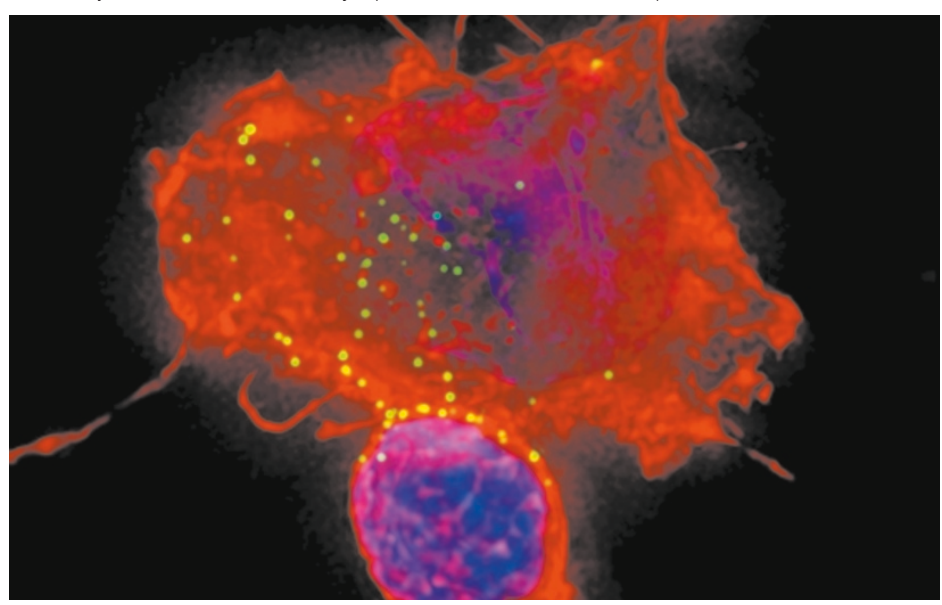

Transfer of HIV (green) from a myeloid dendritic cell to a T cell. Actin (Phalloidin staining) is shown in red and DNA (Hoechst staining) is shown in blue. Image courtesy of D. McDonald and T. Hope, University of Illinois at the Chicago College of Medicine, USA.

\section{IN BRIEF}

\section{CELLULAR MICROBIOLOGY}

\section{Pathogenic bacteria attach to human fibronectin} through a tandem $\beta$-zipper.

Schwarz-Linek, U. et al. Nature 423, 177-181 (2003)

These authors used a structural approach to understand bacterial fibronectin-binding protein (FnBP)-mediated adhesion to host cells. They determined the structure of a streptococcal FnBP peptide (B3) in complex with modules 1 and $2\left({ }^{1} \mathrm{~F} 1{ }^{2} \mathrm{~F} 1\right)$ of the amino-terminal, bacterium-binding site of host-cell fibronectin. The interaction seen is the first example of a tandem $\beta$-zipper, in which $\beta$-strands form on adjacent domains (in this case, the sequential F1 modules).

\section{MOLECULAR MOTORS}

Mutations in dynein link motor neuron degeneration to defects in retrograde transport.

Hafezparast, M. et al. Science 300, 808-812 (2003)

The underlying genetic defects of many motor neuron diseases, including most amyotrophic lateral sclerosis (ALS) cases, are unknown. But, Hafezparast et al. now show that missense point mutations in the cytoplasmic dynein heavy chain 1 (Dnchc1) gene cause progressive motor neuron degeneration in heterozygous mice, and that homozygous mice present with Lewy-like inclusion bodies. Dnchc1 mutations are thought to impair fast retrograde transport in spinal-cord motor neurons.

\section{SIGNAL TRANSDUCTION}

Switch-of-function mutants based on morphology classification of Ras superfamily small GTPases.

Do Heo, W. \& Meyer, T. Cell 113, 315-328 (2003)

Proteins from the same family can have very different functional roles. Indeed, these authors found that cell morphology changes induced by constitutively active small GTPases could be grouped into nine classes. To find out which amino acids in a functional class are relevant for functional specificity, they developed a 'switch-ofclass' algorithm, which was validated experimentally. Surprisingly, residues that define morphology were mostly outside the known interaction surfaces and were structurally far apart from each other.

\section{CELL ADHESION}

\section{Rap1 translates chemokine signals to integrin}

activation, cell polarization, and motility across vascular endothelium under flow.

Shimonaka, M. et al. J. Cell Biol. 161, 417-427 (2003)

Lymphocytes migrate in response to chemokines in a process that requires adhesive interactions with the vascular endothelium. Shimonaka et al. now show that the small GTPase Rap1 is crucially involved in the chemokine-mediated rapid increase of the adhesive activity of two integrins to immobilized intercellular adhesion molecule-1 (ICAM-1). Rap1 also causes increased cell migration and cell polarization in lymphocytes. 